\title{
Scintillator performance considerations for dedicated breast computed tomography
}

Srinivasan Vedantham, Linxi Shi, Andrew Karellas

Srinivasan Vedantham, Linxi Shi, Andrew Karellas, "Scintillator performance considerations for dedicated breast computed tomography," Proc. SPIE 10393, Radiation Detectors in Medicine, Industry, and National Security XVIII, 103930M (14 September 2017); doi: 10.1117/12.2279225

EDent: SPIE Optical Engineering + Applications, 2017, San Diego, California, United States 


\title{
Scintillator performance considerations for dedicated breast computed tomography
}

\author{
Srinivasan Vedantham*a ${ }^{\text {a }}$ Linxi Shi ${ }^{\mathrm{b}}$, Andrew Karellas ${ }^{\mathrm{a}}$ \\ ${ }^{a}$ Department of Medical Imaging, University of Arizona, Tucson, AZ, USA 85724; \\ ${ }^{\mathrm{b}}$ Nuclear and Radiological Engineering and Medical Physics Programs, The George W. Woodruff \\ School of Mechanical Engineering, Georgia Institute of Technology, Atlanta, GA, USA 30332
}

\begin{abstract}
Dedicated breast computed tomography (BCT) is an emerging clinical modality that can eliminate tissue superposition and has the potential for improved sensitivity and specificity for breast cancer detection and diagnosis. It is performed without physical compression of the breast. Most of the dedicated BCT systems use large-area detectors operating in cone-beam geometry and are referred to as cone-beam breast CT (CBBCT) systems. The large-area detectors in CBBCT systems are energy-integrating, indirect-type detectors employing a scintillator that converts $\mathrm{x}$-ray photons to light, followed by detection of optical photons. A key consideration that determines the image quality achieved by such CBBCT systems is the choice of scintillator and its performance characteristics. In this work, a framework for analyzing the impact of the scintillator on CBBCT performance and its use for task-specific optimization of CBBCT imaging performance is described.
\end{abstract}

Keywords: computed tomography (CT), cone-beam CT (CBCT), dedicated breast computed tomography (BCT), breast cancer, scintillator

\section{INTRODUCTION}

Digital mammography, which has replaced screen-film mammography, is now the primary imaging modality for breast cancer screening and diagnosis [1-4]. However, superposition of tissue generates unnecessary recalls for additional imaging or results in missed cancers. Digital breast tomosynthesis (DBT), which is a limited-angle tomography, was developed to partially overcome this problem [5-9] and is being rapidly adopted for clinical imaging. While DBT has demonstrated reduction in false-positive recall rate and improvement in cancer detection rate, visualization of lesions in extremely dense breasts continues to be a challenge [10]. Both digital mammography and DBT are performed with vigorous breast compression. In comparison, dedicated breast computed tomography (BCT) does not require physical compression of the breast. Dedicated BCT eliminates tissue superposition as demonstrated by reduced anatomical noise [11-13] and multi-planar reconstructions allow for visualizing the breast in any orientation in addition to standard anatomical planes. Dedicated BCT is inherently quantitative allowing for determination of volumetric breast density [14] for breast cancer risk estimation and contrast-enhanced BCT with intravenously administered contrast media can allow for better discrimination of malignant and benign lesions [15]. At radiation dose similar to diagnostic workup with mammography [16], dedicated BCT demonstrated improved sensitivity than mammography-based diagnostic imaging [17] and this study led to the US Food and Drug Administration (FDA) approval for non-contrast diagnostic use.

The motivation for this study was that an early study with dedicated BCT operating at radiation dose similar to standard 2-view mammography, reported improved conspicuity for soft tissue lesions but reduced conspicuity for microcalcifications [18]. Since then, there have been substantial technological advances in hardware, such as the availability of x-ray tubes with focal spot size similar to that used for screening mammography, and high-resolution, low-noise x-ray detectors. Most of the dedicated BCT systems use cone-beam geometry with large-area, indirect-type detectors that employ a scintillator to convert x-ray photons to light, followed by detection of optical photons [18-22]. For these cone-beam breast CT (CBBCT) systems, the choice of scintillator and its thickness impact the overall imaging performance. Hence, in this work a framework for analyzing the impact of the scintillator and task-specific optimization of its thickness for CBBCT imaging are described.

*svedantham@radiology.arizona.edu; phone 1520 626-6641; fax 1520 626-7093; medicine.arizona.edu

Radiation Detectors in Medicine, Industry, and National Security XVIII, edited by Gary P. Grim,

Lars R. Furenlid, H. Bradford Barber, Proc. of SPIE Vol. 10393, 103930M · C 2017 SPIE

CCC code: $0277-786 X / 17 / \$ 18 \cdot$ doi: $10.1117 / 12.2279225$ 


\section{METHODS}

\subsection{System description}

Most CBBCT systems and clinical prototypes utilize a CsI:Tl scintillator coupled amorphous silicon flat-panel detector (PaxScan 4030CB, Varex Imaging, Salt Lake City, UT) with a native pixel pitch of $194 \mu \mathrm{m}$ that is operated at $388 \mu \mathrm{m}$, by $2 \times 2$ pixel binning [14-20]. Since high-resolution, low-noise active-pixel complementary metal-oxide semiconductor (CMOS) sensors with $75 \mu \mathrm{m}$ native pixel pitch (Dexela CMOS 2923CL, Varex Imaging, Salt Lake City, UT) are now available and can be potentially beneficial for imaging microcalcifications, such a detector was modeled in this work. In order to minimize the likelihood for patient motion, $2 \times 2$ pixel binning for $150 \mu \mathrm{m}$ pixel pitch was considered, so that the scan time could be less than 5 seconds. This CMOS detector features a dead-space (inactive region) at the chest-wall edge of the detector of $3 \mathrm{~mm}$ similar to digital mammography, compared to $34.2 \mathrm{~mm}$ in the PaxScan 4030CB detector used in the US FDA approved CBBCT system. The reduced dead-space at the chest-wall can improve posterior coverage $[14,22]$. The imaging geometry from a clinical prototype CBBCT system currently used for in vivo human trials was replicated. The distance from the axis of rotation, also referred to as isocenter in literature, to the source $(\mathrm{SCD})$ is $634 \mathrm{~mm}$ and the source-to-detector distance is $907 \mathrm{~mm}$. For this study, 300 projections over $2 \pi\left(360^{\circ}\right)$ circular $\mathrm{x}$-ray source trajectory was considered to be consistent with the acquisition protocol used in the US FDA approved CBBCT system [19-21]. For breast CT, multiple studies have indicated that the optimal x-ray tube voltage to be in the range of 55-65 kV [23-25]. For this study, an x-ray tube with a $0.3 \mathrm{~mm}$ (nominal) focal spot size (M-1583, Varian Medical Systems) operating at $60 \mathrm{kV}$ and filtered with $0.15 \mathrm{~mm}$ of $\mathrm{Cu}$ and $1 \mathrm{~mm}$ of Al providing a $1^{\text {st }}$ half-value layer thickness of $3.68 \mathrm{~mm}$ of $\mathrm{Al}$ was considered. The x-ray source, detector and acquisition parameters for the CBBCT system modeled are included in Table 1.

Table 1. Acquisition parameters, x-ray source and detector specifications for the cone-beam breast CT system (CBBCT) employing an active pixel CMOS detector.

\begin{tabular}{|l|c|}
\hline Parameters/Specifications & CBBCT \\
\hline Imaging geometry & $634 \mathrm{~mm}$ \\
\hline Source-to-isocenter distance (SCD) & $907 \mathrm{~mm}$ \\
\hline Source-to-detector distance (SDD) & $0.3 \mathrm{~mm}$ \\
\hline$X$-ray source & 60 \\
\hline Focal spot & $\mathrm{W}$ \\
\hline KV & $0.15 \mathrm{~mm} \mathrm{Cu}+$ \\
\hline Anode & $1 \mathrm{~mm} \mathrm{Al}$ \\
\hline X-ray beam filtration & 3.68 \\
\hline $1^{\text {st }}$ Half-value thickness (mm of Al) & $75 \mu \mathrm{m}$ \\
\hline$X$-ray detector & $2 \times 2$ \\
\hline Native pixel pitch & 0.84 \\
\hline Pixel binning & $362 \mathrm{e}-$ \\
\hline Fill factor & \multicolumn{2}{|c|}{} \\
\hline CsI:Tl thickness & 300 \\
\hline System noise & $4.5 \mathrm{mGy}$ \\
\hline Scan parameters & FBP (ramp) \\
\hline Number of projections & $\mu \mathrm{m}, 75 \mu \mathrm{m}$ increments \\
\hline Dose limit & \\
\hline Reconstruction & \\
\hline
\end{tabular}




\subsection{Scintillator}

Columnar thallium-doped cesium iodide (CsI:Tl) is now the scintillator of choice for medical x-ray imaging and has mostly replaced granular gadolinium oxysulfide (Gadox) due to the improved spatial resolution properties of CsI:Tl that are grown in needle-like columns of approximately 3-5 $\mu \mathrm{m}$ diameter. Also, granular noise caused by grain-size variations in Gadox scintillators that contribute to variability in light output are not of concern with CsI:Tl. CsI:Tl has a density of $4.51 \mathrm{~g} / \mathrm{cm}^{3}$ and emits approximately 54,000 optical photons per absorbed 1-MeV x-ray photon with an emission peak at approximately $540 \mathrm{~nm}$ [26]. In this work, 300-600 $\mu \mathrm{m}$ thick CsI:Tl scintillators, in increments of 75 $\mu \mathrm{m}$, deposited on an amorphous carbon substrate (Hamamatsu Corp., Bridgewater, NJ), which were previously evaluated were considered [27]. In order to protect the CMOS detector from direct x-ray interaction while transmitting the optical photons, a fiberoptic plate is required. An 1-inch thick radiation-hard fiberoptic plate with known refractive indices for the core and the clad was considered based on a prior study [28] and was assumed to be directly bonded to the CMOS sensor. Optical coupling media to match the refractive refractive index was assumed between the scintillator and the fiberoptic plate and the optical coupling efficiency was analytically determined $[29,30]$.

\subsection{Breast model and radiation dose}

The pendant breast was modeled as a semi-ellipsoid with diameter at the chest-wall of $14 \mathrm{~cm}$ and chest-wall to nipple length of $10.5 \mathrm{~cm}$ that corresponds to an average sized breast [14]. The breast was assumed to be centered in the CBBCT field of view and co-aligned with the axis of rotation. Although the mean fibroglandular weight fraction has been determined to be approximately $15 \%$ [14], fibroglandular weight fraction of $50 \%$ was used to be consistent with the breast dosimetry protocol in the United States. The conversion factors that are needed to determine the average glandular dose (AGD) to the breast from measurements of air kerma at the isocenter were obtained from prior Monte Carlo simulations using the GEANT4 toolkit [31]. In order to explore the potential of CBBCT for breast cancer screening, the AGD to the breast was limited to $4.5 \mathrm{mGy}$, which is approximately equivalent to 2-view mammography for an average breast. It is relevant to note that the goal of breast CT is to replace the 2-standard mammography views with a single scan and the reconstructed image can be visualized in any orientation.

\subsection{Image reconstruction}

The projection dataset was assumed to be reconstructed using a ramp-filtered backprojection algorithm with bilinear interpolation. No apodization filters were assumed. The reconstructed voxel pitch was $105 \mu \mathrm{m}$, determined as the ratio of the detector pixel pitch $(150 \mu \mathrm{m})$ to the system magnification. The system magnification was 1.43 and defined as the ratio of the SDD $(907 \mathrm{~mm})$ to the $\mathrm{SCD}(634 \mathrm{~mm})$.

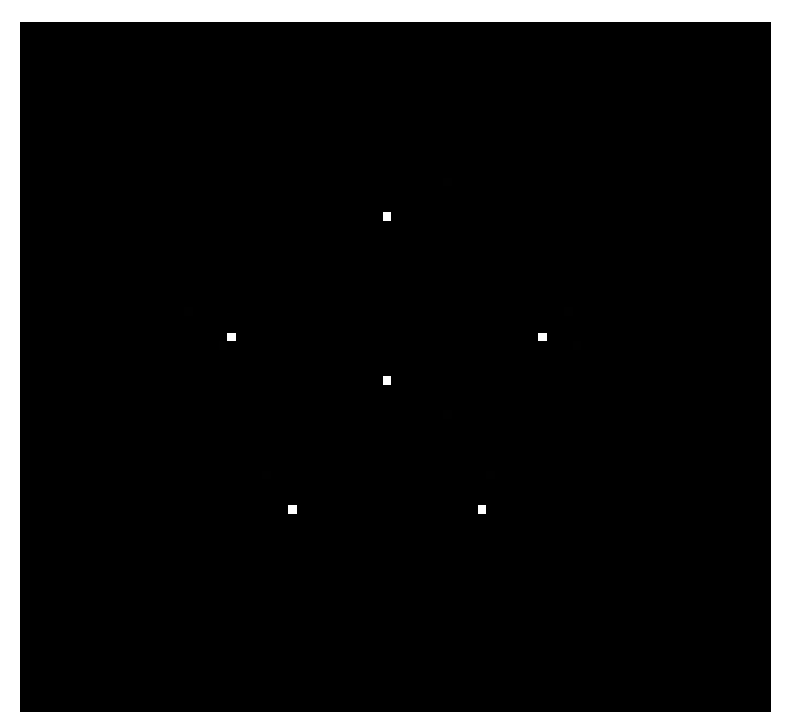

Figure 1. The imaging task considered for the study. The microcalcification cluster was modeled as $220 \mu \mathrm{m}$ calcium carbonate $\left(\mathrm{CaCO}_{3}\right)$ spheres arranged as a pentagon with $2 \mathrm{~mm}$ spacing on the sides, similar to that in the American College of Radiology (ACR) recommended phantom for mammography accreditation. 


\subsection{Task function}

A prior study reported that conspicuity for microcalcifications is limited at AGD equivalent to standard 2-view mammography [18]. Since soft tissue lesions are readily visualized, the task of detecting a microcalcification cluster was chosen for this study. The microcalcification cluster was modeled as $220 \mu \mathrm{m}$ calcium carbonate $\left(\mathrm{CaCO}_{3}\right)$ spheres arranged as a pentagon with $2 \mathrm{~mm}$ spacing on the sides, similar to that in the American College of Radiology (ACR) recommended phantom for mammography accreditation and is shown in Figure 1. The calcification cluster was assumed to be located in the coronal plane (parallel to the chest-wall) and at the intersection of the axis of rotation with the central ray, defined as the ray with $0^{\circ}$ cone and fan angles. The viewing protocol used in ongoing clinical studies is scrolling average intensity projection (AvIP) of 10 slices. Hence, the task function and objective metrics were computed as 10 slice average.

\subsection{Detectability index}

For the specified breast dimensions, imaging geometry and acquisition parameters, the air kerma at isocenter in each projection view and consequently the fluence incident on the breast were determined. The x-ray fluence transmitted through the breast was propagated through the imaging chain using a validated parallel-cascaded systems model $[27,30$, $32,33]$ that accounted for characteristic $\mathrm{x}$-ray emission and reabsorption within the scintillator. The model was used to obtain the objective imaging metrics in projection space such as modulation transfer function (MTF), noise power spectrum (NPS), noise-equivalent quanta (NEQ), and detective quantum efficiency (DQE) in spatial frequency (Fourier) domain [33]. After log-normalization, ramp-filtered backprojection with bilinear interpolation was used to extend the model to reconstructed object space [34]. The obtained objective metrics were weighted with the task function to determine the International Commission on Radiation Units (ICRU) recommended detectability index $(d$ ) using nonprewhitening numerical observer with eye filter (NPWE). The CsI:Tl thickness that maximized $d^{\prime}$ was considered optimal for CBBCT.

\section{RESULTS}

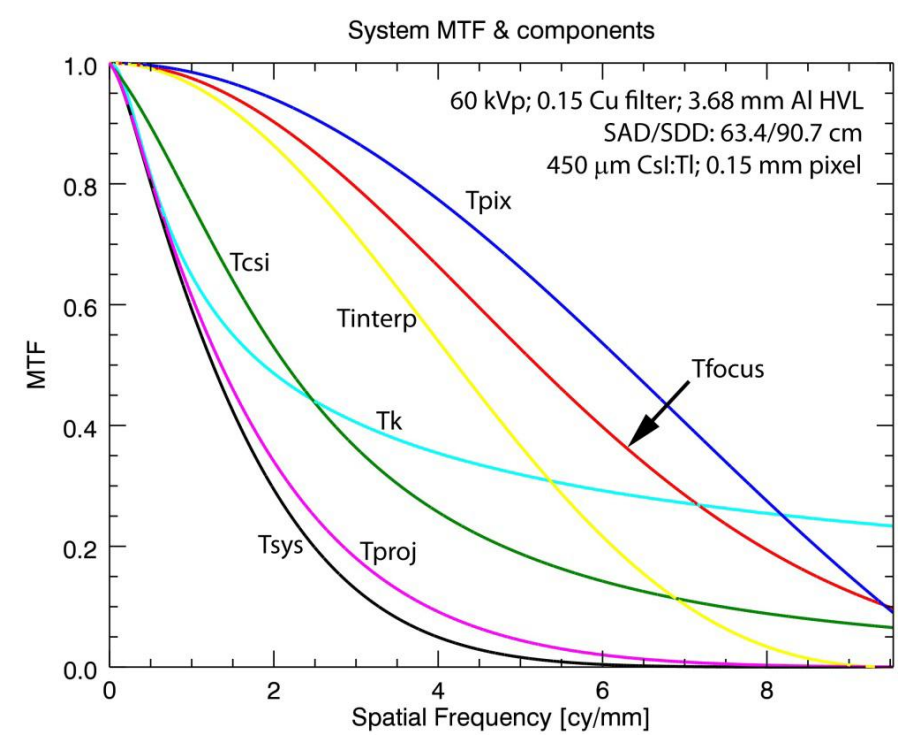

Figure 2. Modulation transfer function (MTF) of a CBBCT system using a CMOS detector with $450 \mu \mathrm{m}$ thick CsI:Tl scintillator. The overall system MTF in the reconstructed object space $\left(\mathrm{T}_{\text {sys }}\right)$ is dependent on the projection space MTF $\left(\mathrm{T}_{\text {proj }}\right)$ and the bilinear interpolation $\left(\mathrm{T}_{\text {interp }}\right)$ used during image reconstruction. The projection space MTF $\left(\mathrm{T}_{\text {proj }}\right)$ is influenced by the $\mathrm{x}$-ray focal spot associated blur $\left(\mathrm{T}_{\text {focus }}\right)$, the blur associated with the proportion of $\mathrm{x}$-ray photons that undergo characteristic x-ray emission and reabsorption $\left(\mathrm{T}_{\mathrm{k}}\right)$, the optical blur associated with the CsI:Tl scintillator $\left(\mathrm{T}_{\mathrm{csi}}\right)$ and the pixel aperture function $\left(\mathrm{T}_{\mathrm{pix}}\right)$. 
Figure 2 shows the modulation transfer function (MTF) of the CBBCT system in projection space $\left(\mathrm{T}_{\text {proj }}\right)$ and the overall system MTF $\left(\mathrm{T}_{\text {sys }}\right)$ in reconstructed object space along with the individual components that contribute to the system MTF. Key image acquisition and imaging geometry parameters are included in the figure legend. The overall system MTF in the reconstructed object space $\left(\mathrm{T}_{\text {sys }}\right)$ is dependent on the projection space MTF $\left(\mathrm{T}_{\text {proj }}\right)$ and the bilinear interpolation ( $\mathrm{T}_{\text {interp }}$ ) used during image reconstruction. The projection space MTF $\left(\mathrm{T}_{\text {proj }}\right)$ is influenced by the x-ray focal spot associated blur $\left(\mathrm{T}_{\text {focus }}\right)$, the blur associated with the proportion of $\mathrm{x}$-ray photons that undergo characteristic $\mathrm{x}$-ray emission and reabsorption $\left(\mathrm{T}_{\mathrm{k}}\right)$, the optical blur associated with the CsI:Tl scintillator $\left(\mathrm{T}_{\mathrm{csi}}\right)$ and the pixel aperture function $\left(T_{\text {pix }}\right)$. The limiting resolution ( $10 \%$ system MTF) determined by the model was 3.46 cycles $/ \mathrm{mm}$. An independent report using a similar detector with $450 \mu \mathrm{m} \mathrm{CsI:Tl} \mathrm{thickness} \mathrm{operating} \mathrm{at} 60 \mathrm{kV}$ and with a slightly different imaging geometry reported a limiting resolution of $3.5 \mathrm{cycles} / \mathrm{mm}$ [35]. Hence, the overall model is in good agreement with empirical measurements.

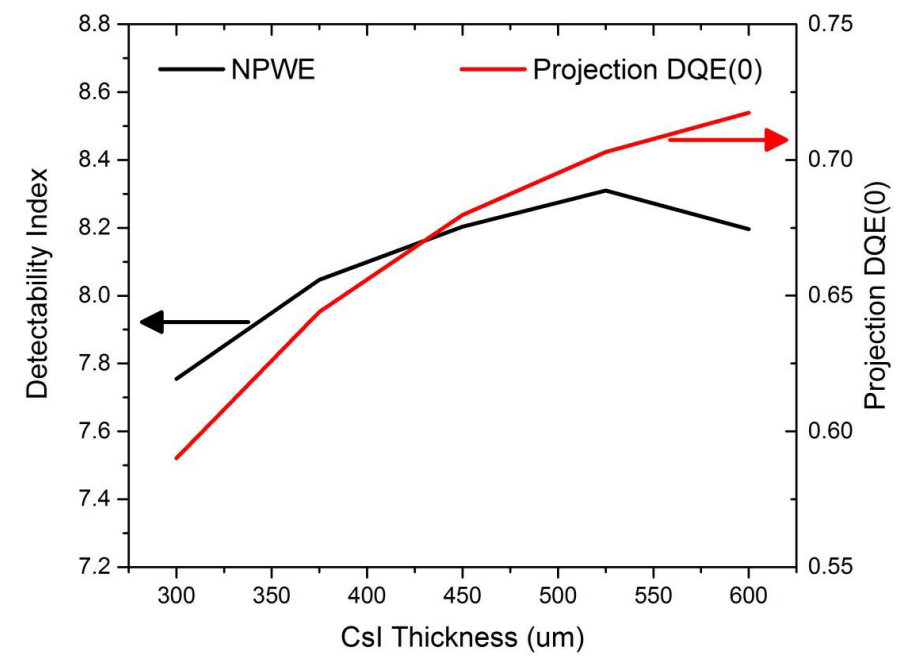

Figure 3. The projection space zero-spatial frequency DQE, DQE(0) (right y-axis), and the detectability index determined using NPWE numerical observer for the detection of microcalcification cluster (left y-axis) are shown as a function of CsI:Tl scintillator thickness. $\mathrm{DQE}(0)$ shows a monotonic increasing trend with CsI:Tl thickness due to increased quantum efficiency, whereas the detectability index shows a peak at $525 \mu \mathrm{m}$.

Figure 3 shows the projection space zero-spatial frequency DQE (right y-axis) as a function of scintillator thickness. There is an monotonic increase in zero-frequency DQE, DQE(0), with increasing CsI:Tl thickness that is consistent with the expectation of improved quantum efficiency. The detectability index $\left(d^{\prime}\right)$ determined using NPWE observer model as a function of scintillator thickness is also shown (left y-axis). The detectability index shows a peak at $525 \mu \mathrm{m}$ CsI:Tl thickness. For CsI:Tl thickness less than $525 \mu \mathrm{m}$, the improvement in detectability index can be attributed to improved quantum efficiency and zero-frequency DQE. However, when the CsI:Tl thickness is increased beyond $525 \mu \mathrm{m}$, the increased optical blur for thicker scintillators contributes to reduction in detectability index.

\section{CONCLUSION}

At radiation dose equivalent to standard 2-view mammography and for the task of detecting the specified microcalcification cluster, $525 \mu \mathrm{m}$ thick CsI:Tl scintillator is theoretically optimal for dedicated cone-beam breast CT. It is relevant to note that the standard CsI:Tl thickness provided by the detector manufacturer is either $150 \mu \mathrm{m}$ or $600 \mu \mathrm{m}$ and it likely to be of benefit to modify this thickness for the intended CBBCT application. The observations from this study needs to be empirically validated. Importantly, the study provides a framework that can be adapted for other imaging tasks and would enable task-specific determination and optimization of acquisition and reconstruction parameters. 


\section{ACKNOWLEDGEMENTS}

This work was supported in part by the National Cancer Institute (NCI) of the National Institutes of Health (NIH) grants R01 CA195512, R01 CA199044 and R21 CA134128. The contents are solely the responsibility of the authors and do not reflect the official views of the NCI or the NIH.

\section{REFERENCES}

[1] E. D. Pisano, C. Gatsonis, E. Hendrick et al., "Diagnostic performance of digital versus film mammography for breast-cancer screening," N Engl J Med, 353(17), 1773-83 (2005).

[2] S. Vedantham, A. Karellas, S. Suryanarayanan et al., "Full breast digital mammography with an amorphous silicon-based flat panel detector: physical characteristics of a clinical prototype," Med Phys, 27(3), 558-67 (2000).

[3] S. Suryanarayanan, A. Karellas, S. Vedantham et al., "Flat-panel digital mammography system: contrast-detail comparison between screen-film radiographs and hard-copy images," Radiology, 225(3), 801-7 (2002).

[4] S. Suryanarayanan, A. Karellas, and S. Vedantham, "Physical characteristics of a full-field digital mammography system," Nuclear Instruments \& Methods in Physics Research Section a-Accelerators Spectrometers Detectors and Associated Equipment, 533(3), 560-570 (2004).

[5] L. T. Niklason, B. T. Christian, L. E. Niklason et al., "Digital tomosynthesis in breast imaging," Radiology, 205(2), 399-406 (1997).

[6] S. Suryanarayanan, A. Karellas, S. Vedantham et al., "Comparison of tomosynthesis methods used with digital mammography," Acad Radiol, 7(12), 1085-97 (2000).

[7] S. Suryanarayanan, A. Karellas, S. Vedantham et al., "Evaluation of linear and nonlinear tomosynthetic reconstruction methods in digital mammography," Acad Radiol, 8(3), 219-24 (2001).

[8] I. Sechopoulos, S. Suryanarayanan, S. Vedantham et al., "Scatter radiation in digital tomosynthesis of the breast," Med Phys, 34(2), 564-76 (2007).

[9] I. Sechopoulos, S. Suryanarayanan, S. Vedantham et al., "Computation of the glandular radiation dose in digital tomosynthesis of the breast," Med Phys, 34(1), 221-32 (2007).

[10] S. Vedantham, A. Karellas, G. R. Vijayaraghavan et al., "Digital Breast Tomosynthesis: State of the Art," Radiology, 277(3), 663-84 (2015).

[11] S. Vedantham, L. Shi, A. Karellas et al., "Dedicated breast CT: anatomic power spectrum." The Second International Conference on Image Formation in X-ray Computed Tomography, Fort Douglas/Salt Lake City, UT. Proceedings of the 2nd International Conference on Image Formation in X-ray Computed Tomography, 70-73 (2012 Published).

[12] K. G. Metheany, C. K. Abbey, N. Packard et al., "Characterizing anatomical variability in breast CT images," Med Phys, 35(10), 4685-94 (2008).

[13] S. Vedantham, L. Shi, S. J. Glick et al., "Scaling-law for the energy dependence of anatomic power spectrum in dedicated breast CT," Med Phys, 40(1), 011901 (2013).

[14] S. Vedantham, L. Shi, A. Karellas et al., "Dedicated breast CT: fibroglandular volume measurements in a diagnostic population," Med Phys, 39(12), 7317-28 (2012).

[15] N. D. Prionas, K. K. Lindfors, S. Ray et al., "Contrast-enhanced dedicated breast CT: initial clinical experience," Radiology, 256(3), 714-23 (2010).

[16] S. Vedantham, L. Shi, A. Karellas et al., "Personalized estimates of radiation dose from dedicated breast CT in a diagnostic population and comparison with diagnostic mammography," Phys Med Biol, 58(22), 7921-36 (2013).

[17] E. B. Cole, A. S. Campbell, S. Vedantham et al., "Clinical Performance of Dedicated Breast Computed Tomography in Comparison to Diagnostic Digital Mammography [abstract \# SSA01-09]." 101st Scientific Assembly and Annual Meeting of the Radiological Society of North America (RSNA 2015), Chicago, IL (2015 Published).

[18] K. K. Lindfors, J. M. Boone, T. R. Nelson et al., "Dedicated breast CT: initial clinical experience," Radiology, 246(3), 725-33 (2008).

[19] A. M. O'Connell, A. Karellas, and S. Vedantham, "The potential role of dedicated 3D breast CT as a diagnostic tool: review and early clinical examples," Breast J, 20(6), 592-605 (2014). 
[20] S. Vedantham, A. M. O'Connell, L. Shi et al., "Dedicated Breast CT: Feasibility for Monitoring Neoadjuvant Chemotherapy Treatment," J Clin Imaging Sci, 4, 64 (2014).

[21] L. Shi, S. Vedantham, A. Karellas et al., "Skin thickness measurements using high-resolution flat-panel conebeam dedicated breast CT," Med Phys, 40(3), 031913 (2013).

[22] S. Vedantham, A. Karellas, M. M. Emmons et al., "Dedicated breast CT: geometric design considerations to maximize posterior breast coverage," Phys Med Biol, 58(12), 4099-118 (2013).

[23] S. J. Glick, S. Vedantham, and A. Karellas, "Investigation of optimal kVp settings for CT Mammography using a Flat-panel Imager." Medical Imaging 2002: Physics of Medical Imaging, 4682, 392-402 (2002 Published).

[24] N. D. Prionas, S. Y. Huang, and J. M. Boone, "Experimentally determined spectral optimization for dedicated breast computed tomography," Med Phys, 38(2), 646-55 (2011).

[25] S. J. Glick, S. Thacker, X. Gong et al., "Evaluating the impact of X-ray spectral shape on image quality in flatpanel CT breast imaging," Med Phys, 34(1), 5-24 (2007).

[26] V. V. Nagarkar, S. V. Tipnis, V. B. Gaysinskiy et al., "New Design of a Structured CsI(Tl) Screen for Digital Mammography." Proc SPIE 5030, 541-546.

[27] S. Vedantham, A. Karellas, S. Suryanarayanan et al., "Solid-state fluoroscopic imager for high-resolution angiography: physical characteristics of an $8 \mathrm{~cm}$ x $8 \mathrm{~cm}$ experimental prototype," Med Phys, 31(6), 1462-72 (2004).

[28] S. Vedantham, A. Karellas, and S. Suryanarayanan, "Attenuation characteristics of fiberoptic plates for digital mammography and other X-ray imaging applications," J Xray Sci Technol, 11(4), 219-30 (2003).

[29] S. Hejazi, and D. P. Trauernicht, "System considerations in CCD-based x-ray imaging for digital chest radiography and digital mammography," Med Phys, 24(2), 287-97 (1997).

[30] S. Suryanarayanan, A. Karellas, S. Vedantham et al., "Theoretical analysis of high-resolution digital mammography," Phys Med Biol, 51(12), 3041-55 (2006).

[31] S. Vedantham, L. Shi, A. Karellas et al., "Dedicated breast CT: radiation dose for circle-plus-line trajectory," Med Phys, 39(3), 1530-41 (2012).

[32] S. Vedantham, and A. Karellas, "Modeling the performance characteristics of computed radiography (CR) systems," IEEE Trans Med Imaging, 29(3), 790-806 (2010).

[33] S. Vedantham, A. Karellas, and S. Suryanarayanan, "Solid-state fluoroscopic imager for high-resolution angiography: parallel-cascaded linear systems analysis," Med Phys, 31(5), 1258-68 (2004).

[34] D. J. Tward, and J. H. Siewerdsen, "Noise aliasing and the 3D NEQ of flat-panel cone-beam CT: effect of 2D/3D apertures and sampling," Med Phys, 36(8), 3830-43 (2009).

[35] P. M. Gazi, K. Yang, G. W. Burkett, Jr. et al., "Evolution of spatial resolution in breast CT at UC Davis," Med Phys, 42(4), 1973-81 (2015). 